\title{
Study on ESD Protection Design of Electronic Communication Products
}

\author{
Meifang Cai ${ }^{1}$ \\ ${ }^{1}$ Nanchang Institute of Technology, Nanchang, Jiang Xi, 330108 \\ 346591653@163.com
}

\section{KEYWORDS: ESD; Communication Products; Protection Design}

\begin{abstract}
This article is to introduce the harmless of ESD electronic communications products, protective measures to prevent adverse reactions buildup of static electricity caused by ESD, electronic communication products for electrostatic discharge is undoubtedly an invisible killer, electronic communication products in use. If the process is electrostatic discharge interference will not only affect the normal operation of the product, but also cause some degree of damage to electronic components, electronic products, even in the case of a special cause unexpected accidents, electronic communication products in the design, trial, production process and operation, there is often electrostatic discharge, so we want to increase the protection of electronic communication products design, analysis from different aspects of electronic products from electrostatic discharge harm, for the moment, saying there are many protective measures questions introduces electronic communication products ESD protection design should pay attention to the problem, as well as the basic requirements of the protection of electronic communications products.
\end{abstract}

\section{The Disadvantage of ESD Electronic Product harm}

ESD is a discharge of static electricity, and is a fast charge phenomenon, which is after the electronic components into the static electricity generated by the power supply has been induced to produce rapid discharge phenomenon, and ESD protection is to prevent the accumulation of static electricity caused explosion electric shock and other accidents, the main principles of protection is to inhibit the generation of static electricity, static electricity leak rate of acceleration, or electrostatic integrated, electronic products and body itself is very easy to produce static electricity, electrical contacts at each other design quality communications products indirectly affect the rate of qualified products in the production process equipment, can also cause internal disputes between shirk responsibility, to a certain extent, dealers and products and equipment owned by the user will be varying degrees of impact, and these problems will directly affect the product's position in the market and the impact of reported damage electronic components caused by electrostatic discharge accounted for $60 \%$ of the electronic devices, so the electronic communication products ESD protection design throughout the design of electronic products is an indispensable part, protection design deficiencies .EOS of confusion for EOS and ESD is, ESD is part of a special class, but ESD and EOS often will confuse the performance index for protection of the design, the EOS wrong means work performed as ESD products, the type of identification system identification system for determining the ESD protection system plays a key role, different types of systems to the requirements of protective measures are not the same, if the system of knowledge about the type of recognition is not enough, so out of the protection system design is unreasonable, the sensitivity of different systems for ESD is not the same, the main sensitive types of voltage-sensitive sensitivity 
and ability, the higher the sensitivity of the assumption that the system voltage, in the protective care will double, if the system is more sensitive to energy, it requires corresponding protection system would loose a lot, the system does not distinguish the voltage-sensitive or sensitive to energy is to be in accordance with the voltage sensitive is to be processed.

ESD cause component failure. When a charged object to form a discharge path through the device or the device itself is charged when a discharge path, ESD will have caused the failure of the device, there is a sudden and complete failure modes and potential failure of slow failure: Sudden total failure, chip dielectric breakdown of the device or burned, one or more electrical parameters suddenly deteriorated and failed completely lost specified functions. It manifests itself in open, short and electrical parameters serious drift, about $10 \%$ probability; potential slow failure, by the ESD device caused minor damage, degradation of performance of the device or parameter index decreased to become problems, so that the circuit work in the future, parameters deteriorated gradually increased, eventually fail, the probability of about $90 \%$.

ESD makes an error message, the logic circuit malfunction tests showed, ESD belong pulse interference on an electronic communication products electrostatic contact discharge, can be measured in a number of positions within the device as the disturbance pulse amplitude several tens of volts, it is this interference pulse, causing the error message, the logic circuit flip, resulting in failure of the product; ESD can produce a band of a few hundred kilohertz tens of $\mathrm{MHz}$, up to the level of electromagnetic pulse interference and when coupled to the electromagnetic pulse interference when sensing circuit products can also cause an error message, resulting in failure of the product.

High-voltage electrostatic adsorption of dust particles easily adsorbed electrostatically charged dust particles, contamination of PCB and the semiconductor chip, so that the insulation resistance is reduced, affecting the device. It can cause serious device failure (for example: CMOS circuit locks).

\section{ESD Protection Design Status of Electronic Communication Products}

Electronic products during ESD protection design according to electrostatic discharge circuit coupled to the electronic coupling methods, such as inductively coupled, as well as directly to the electrostatic capacitance coupling to electronic circuitry coupled way, electrostatic shield, insulate and other operations in order to better electronic communication products ESD protection design, the design includes $\mathrm{E}$ male protection design of electronic communication products cabinet ESD protection design of electronic communication products, metal parts, printed circuit board ESD protection design,

For EOS speaking, ESD is part of a special class, but often designed for protection ESD and EOS will confuse performance indicators, the EOS wrong means work performed as ESD products, identification systems for ESD protection system plays a key role in determining the different types of systems of safeguards requirements are not the same, if the system of knowledge about the type of recognition is not enough, so out of the protection system design is unreasonable, different systems ESD sensitivity is not the same, the main types of voltage-sensitive and sensitive ability sensitivity, high sensitivity of the assumption that the system voltage, in the protective care will double, if the system is more sensitive to energy, that correspond requested protection systems on many loose in the system fail to distinguish between the voltage sensitive or sensitive to energy is to be in accordance with the voltage sensitive to treatment, to ensure the integrity of the ESD protection. 


\section{Redesign of ESD Protection Scheme}

Clear Level ESD protection scheme. ESD protection consists of two levels: basic protection and comprehensive protection. ESD, basic protection program includes a conductive working surface ground personnel grounding wrist strap (1M $\Omega$ resistor to ground), polyethylene protective packaging, personnel $1 \mathrm{~h} \sim 2 \mathrm{~h}$ basic training in the four elements of ESD; ESD, comprehensive protection program needs basic based on the level of additional protective measures. It requires a comprehensive ESD protection device, such as a device for double-wrapped in packaging by adding an outer metal foil packaging. Protection system to be clear is the basis for the work of establishing the level of protection programs. Only determine the level of protection of the system, we can take specific protective measures ESD protection. Establish ESD protection scheme must also comply with industrial, military or standard production enterprises. These standards can provide effective protective measures and concrete steps for the ESD packaging, operation and circuit design. Conduct ESD management. ESD protection program is effective, it is important ESD management. ESD protection program execution management can be determined, personnel training, maintenance cost ESD protection facilities (Cost) situation. Combined with fault diagnosis, you can also get programs to reduce ESD damage Probability of other information, so as to provide reference for the implementation of protection programs. ESD management must be implemented in the course of protection in order to enable all aspects to achieve coordination, so that the program can effectively play a protective role.

Establish systems of protection. Even if all devices have taken anti-ESD measures, there will still be uncertainty on the protection, engineers still need to pass the relevant test to verify the effectiveness of protective measures and procedures, and some additional protective measures even increase ESD the possibility of injury. Get valid data. The ESD protection HBM classified data and engineering practice and equipment linked is very difficult. This is because these devices are only categorized by HBM test, without considering the effects of charged-device model (CDM) and other sources of damage. To ensure the validity and reliability of devices ESD protective measures, the project will use more and more sophisticated means of testing and data analysis methods. As a result, engineers are able to get on ESD problem means more optimized data. Monitor checks include checking the integrity of the ground wire, grounding face, personnel wristband validity. Where examination of the wristband is formed by a strap connected to the low voltage through the body up for testing. The selected low-voltage energy must not be on the device (such as elimination Schottky diode detector diode) induced EOS. If the air ionization device, they need to be checked periodically within the workplace, for two reasons: First, the device may malfunction, not work; the second is the device of ions generated may be insufficient to balance each other, making it a special, quiet power. An experiment with negative ionization on dust control system in the workplace components generated charges, which not only can cause ionization device failure control charged dust particles, but also induced accidents and damage to the operator an electric shock devices.

\section{Conclusion}

In summary, electronic communication products specific ESD protection design needs to consider many aspects, and the product itself has a very specific electronic system complex features. This requires the design of specific electronic communication products, ESD protection, especially in the early stages of the design, the electrostatic protection as an important part of the design of the core, comprehensive ESD protection design, and finally to electronic communications within the 
product design can play a good ESD the effect, effectively prevent static electricity electronic communication products harm.

\section{REFERENCE:}

[1] Liu Hongyu, Li Wentao. Three Anti-Design of Electronic Communications Products to Improve [J]. Henan Institute of Science and Technology: Natural Science Edition, 2010 (3): 104-106.

[2] Tian Hong Wei, Cai Xu. Eccentric Compression Loading .ESD Protection in the Power Converter Unit Assembly Application [J]. Electrical and Mechanical Information, 2013 (9): 116-118.

[3] Feng Xiaojia, Liuyu Kui, Zhu Kunfeng. Structure Design for Bipolar ESD Protection Circuit Failure Analysis [J]. Microelectronics, 2010 (1): 78-80.

[4] Kan De, Peng Cuixia. Research Complex Battlefield Electromagnetic Environment Simulation Technique [J]. Chinese Academy of Electronics Technology, 2011 (6): 163-165.

[5] Luo Muge. Law on Electronic Communications Pastoral Lightning Surge Protection Device Design and Analysis [J]. Guangdong Science And Technology, 2011 (4): 102-103. 